Rapid Reviews COVID-19

\title{
Reviews of "Genetic association analysis of SARS-CoV-2 infection in 455,838 UK Biobank participants"
}

Jonathan Fischer ${ }^{1}$, Giuseppe Novelli ${ }^{2}$

${ }^{1}$ University of Florida, Biostatistics, USA,

${ }^{2}$ Director Lab, University of Rome Tor Vergata: Universita degli Studi di Roma Tor Vergata, Biomedicine and Prevention, Italy

Published on: Dec 09, 2020

DOI: $10.1162 / 2 \mathrm{e} 3983 \mathrm{f} 5.7 \mathrm{fa} 80690$

License: Creative Commons Attribution 4.0 International License (CC-BY 4.0) 
To read the original manuscript, click the link above.

Summary of Reviews: The authors looked for the genetic loci of COVID-19 risk and severity in the UK biobank. Further studies are needed to pinpoint bona fide loci associated with COVID19 severity and susceptibility, though this study method is deemed reliable by reviewers.

\section{Reviewer 1 (Jonathan Fischer) | प्राप् \\ Reviewer 2 (Giuseppe Novelli) |}

\section{RR:C19 Strength of Evidence Scale Key.}

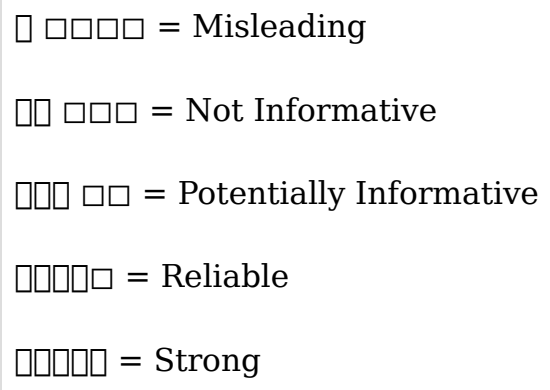

To read the reviews, click the links below. 